\title{
Peering into the cellular cycle
}

\author{
The development of chemical tools and small-molecule inhibitors enables the resolution of critical cellular \\ processes with high spatial and temporal precision.
}

( ells, like all living entities, are born, interact with the surrounding environment and eventually die. The field of cell biology has historical roots in the field of microscopy, enabling visualization of these processes with high resolution. However, approaches to alter cell division, cellular interactions and death, often by genetic and biochemical means, have lagged in their ability to enable spatial and temporal disruption with precision. Chemical biology is well suited to develop improved tools that enable the manipulation of cellular processes with the desired resolution. In this themed issue, we offer a collection of pieces focusing on this intersection of chemical approaches with cell biology and highlighting how this interface may reveal new insights.

Eukaryotic cells are born from a single parental cell through a series of temporally defined phases called mitosis. In particular, the parental cell undergoes a division process producing two daughter cells, each inheriting a single set of chromosomes. Each mitotic phase is regulated by a dynamic and complex set of protein assemblies, and cell biology and chemical tools have been essential in defining the molecular and regulatory basis of these complexes. Given that many cell division events occur at the second or minute time scale, small molecules offer advantages over genetic perturbations in terms of reversibility and temporal control. Early studies utilized general mitotic inhibitors such as colchicine and taxol, which disrupted spindle function and arrested cells at particular mitotic stages. However, these reagents lacked a defined molecular target, limiting their ability to probe the function of an individual mitotic component.

Chen and Lampson discuss recent advances using small molecules to inhibit specific cell cycle regulators that reveal complex interactions not observed with other types of perturbations. One example is apcin, which targets the APC/C E3 ubiquitin ligase complex, which normally promotes mitotic exit. A study of apcin revealed opposing effects through a different regulatory context such as high activity of the spindle assembly checkpoint. In addition, Chen and Lampson describe the development of chemical tools such as optogenetic chemical dimerizers that can direct cell cycle regulators to particular chromosome regions with blue-light exposure. Recent work using these dimerizers to direct the CENP-E kinetochore motor to chromosomes supported a role of CENP-E as a critical motor directing chromosomes to the spindle equator.

During its lifetime, a cell interacts with its environmental surroundings, especially with neighboring cells. Precise cell-to-cell communication is required for many physiological processes such as tissue formation, and this entails complex and transient interactions between cell surface molecules. An earlier Commentary proposed the need for chemical tools to detect, disrupt and reconstitute these intracellular networks. Since that time, advances in microscopy, chemical tagging and cell engineering approaches have enabled progress in this area, summarized in a Review by Bechtel et al. Chemical biology approaches have enabled essential advances; for example, a proximity biotinylation strategy to identify unique peptides secreted by particular cell types, a light-inducible spatial proximity system that modifies proteins on neighboring cells and the use of unnatural amino acid technology to construct a switch for chimeric antigen receptor $\mathrm{T}$ cells that interact with cell surface tumor-specific antigens.

In response to detrimental cellular or environmental conditions, a cell can undergo various forms of death, ranging from apoptosis to ferroptosis, each of which is defined by unique morphological features and molecular markers. Ferroptosis is a non-apoptotic type of cell death that is distinguished by the accumulation of iron-dependent membrane lipid hydroperoxides, resulting in membrane rupture. Given the heavy lipid dependence and involvement of hydroxyl radicals, the study of ferroptosis has been served well by chemical biology approaches. CRISPR-genome screens and lipidomics have identified critical mediators of ferroptosis such as cytochrome P450 oxidoreductase and phosphatidylethanolamines. Mass spectrometry approaches have enabled detection of oxidized phosphatidylethanolamines in ferroptotic cardiomyocytes with improved resolution, while high-throughput small-molecule profiling studies have revealed candidates for chemical modulation. In particular, the use of a high-throughput time-lapse cell death imaging assay enabled profiling of compounds such as rapamycin and PI3K inhibitors that alter ferroptosis directly or indirectly.

Another form of cell death is necroptosis, which is responsive to apoptotic stimuli but does not involve apoptotic regulators such as caspases. Like studies on ferroptosis, chemical biology has helped guide new mechanistic insights and tools for altering necroptosis. One of the first papers published in our journal defined the necroptosis pathway by identification of an inhibitor called necrostatin-1, which targeted the critical regulator RIPK3. RIPK3 recruits the pseudokinase mixed lineage kinase domain-like (MLKL) to necrosomes. The recent generation of monobodies, which are synthetic binding proteins, against specific conformations of MLKL revealed that a conformational transition is needed for MLKL activation.

Damaged cellular organelles and proteins need to be disposed of properly to maintain cellular homeostasis in a process called autophagy. Autophagy involves the engulfment of cellular cargo in a structure called an autophagosome, which fuses with lysosomes resulting in cargo degradation. Whitmarsh-Everiss and Laraia discuss recent developments in identification of activators and inhibitors of distinct steps of this processes. In particular, small-molecule inhibitors that target valosin-containing protein (VCP), an ATPase that initiates autophagy, revealed a novel interaction of VCP with another autophagy protein, Beclin-1.

Despite the advances made, the major and minor moments of a cell's lifetime remain largely hidden and are ripe for discovery. We envision that chemical biology will continue to play a central role in the development of tools and reagents to help further elucidate these hidden and known moments. We hope that the pieces in this issue will inspire and motivate more partnerships that will help drive the field forward and enable new discoveries.

Published online: 25 May 2021

https://doi.org/10.1038/s41589-021-00814-6 